\title{
Crystallization and Melting Behavior of iPP Studied by DSC
}

\author{
GORDANA BOGOEVA-GACEVA, ${ }^{2}$ ACO JANEVSKI, ${ }^{2}$ ANITA GROZDANOV ${ }^{\prime}$ \\ 'Faculty of Technology and Metallurgy, University "St. Ciryl \& Methodius," Skopje, Macedonia \\ ${ }^{2}$ Research Department "OHIS," Skopje, Macedonia
}

Received 27 February 1997; accepted 7 July 1997

\begin{abstract}
This article is a part of a study of model and bulk composites, based on isotactic polypropylene (i-PP) and glass (or carbon) fibers, produced from knitted textile preforms of hybrid yarns. First, we report the results on crystallization and fusion of textile-grade i-PP, used for the processing of hybrid yarns and the corresponding knitted fabrics. The kinetics of the crystallization process, in the dynamic and isothermal regime, was followed by DSC, and the results were analyzed by Avrami, Ozawa, and Harnisch-Muschik methods. Isothermal crystallization of i-PP was carried out at 388$400 \mathrm{~K}$, and values for the Avrami exponent ranging from 1.93 to 4.39 were determined. The equilibrium melting temperature was determined by the Hoffman-Weeks method, and $\gamma=2.54$ was found. Double melting peaks were observed both when the crystallization was performed at lower temperatures (isothermal regime) and at higher cooling rates (nonisothermal regime). A single melting peak appeared upon melting following isothermal crystallization at $400 \mathrm{~K}$. The nonisothermal kinetics data showed that the peak crystallization temperature changes from 377 to $386 \mathrm{~K}$ as the cooling rate decreases from 20 to $3 \mathrm{~K} / \mathrm{min}$. Applying the Ozawa method, a value of the exponent $n$ $=2.33$ was determined, which is in agreement with the results for isothermal crystallization at $391-400 \mathrm{~K}$. The Harnisch-Muschik approach was also applied to compare the results for $n$, and a similar trend in the results of isothermal and nonisothermal crystallization was found, due to the predominant homogeneous mechanism of nucleation at lower cooling rates (lower isothermal $T_{c}$ ) in spite of being heterogeneous at higher cooling rates (higher isothermal $T_{c}$ ). 1998 John Wiley \& Sons, Inc. J Appl Polym Sci 67: 395-404, 1998
\end{abstract}

Key words: polypropylene, isotactic; crystallization; melting behavior; kinetics

\section{INTRODUCTION}

During the last few years, investigations of the melting and crystallization behavior of polypropylene have become a subject of increasing interest. This is mostly due to the development of some new technologies, where thermoplastic polymers are used as matrices for fiber-reinforced composite materials. ${ }^{1-3}$ A new class of thermoplastic composite materials was recently produced based on

Correspondence to: G. Bogoeva-Gaceva.

Journal of Applied Polymer Science, Vol. 67, 395-404 (1998) ○ 1998 John Wiley \& Sons, Inc. innovative cost-effective textile preforms, obtained from hybrid yarns consisting of blended (commingled, cowinded, etc.) reinforcing fibers and thermoplastic filaments. ${ }^{4,5}$

When these textile preforms are converted into composite material by hot pressing, the morphology and the resulting crystallinity of the thermoplastic component may be significantly influenced by the conditions (temperature regime, pressure, etc.) applied during processing. ${ }^{3-5} \mathrm{On}$ the other hand, the degree of crystallinity and morphology of the matrix may, in turn, affect the mechanical properties of the composite material. ${ }^{6.7}$ Also, the crystallization of thermoplastic polymers is often 


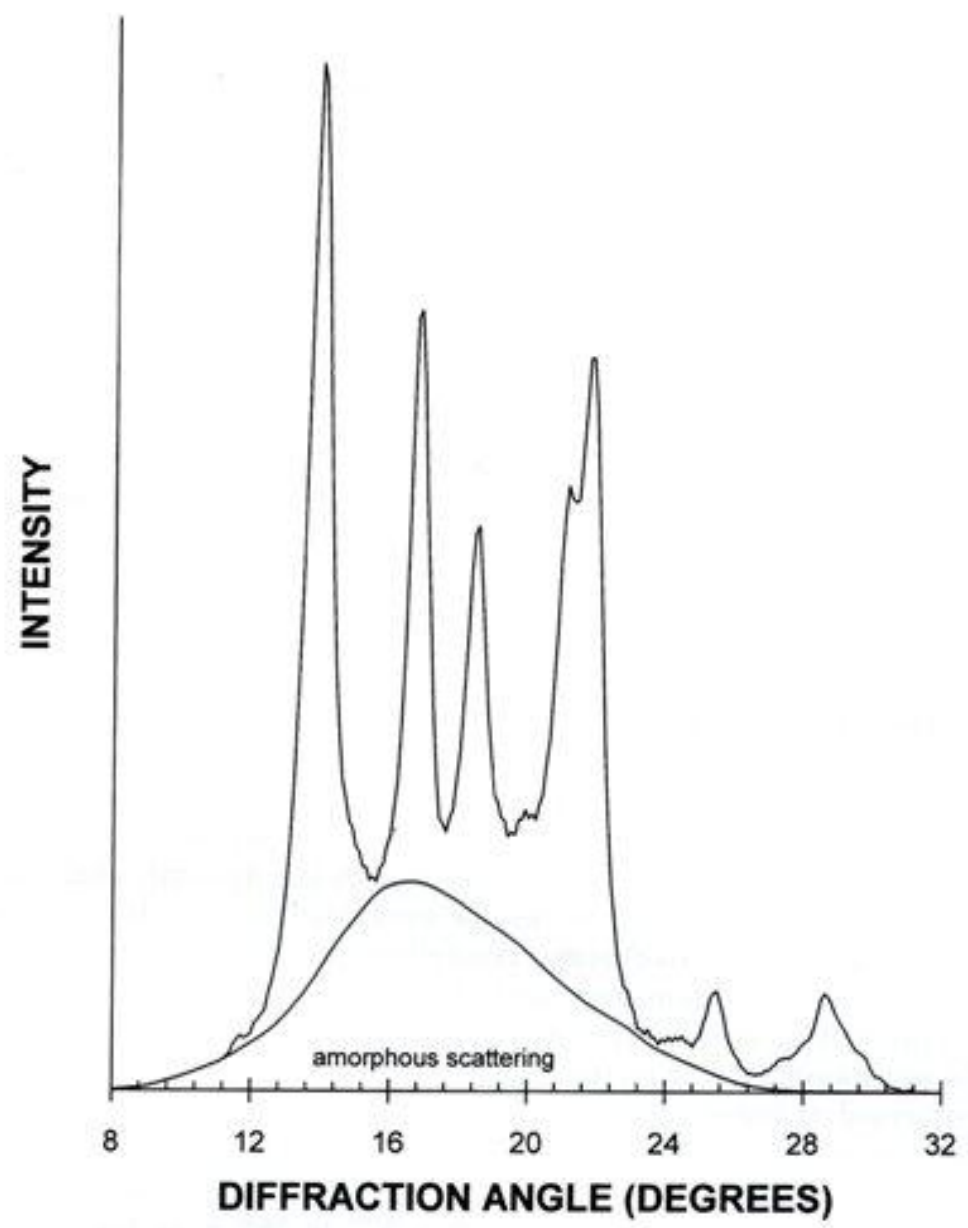

Figure 1 Diffraction pattern of textile-grade i-PP.

found to depend greatly on the possible transcrystallization phenomena. ${ }^{8,9}$

Isotactic polypropylene (i-PP) is a polymorphic material with a high tendency to crystallize in numerous crystal modifications, such as monoclinic $(\alpha)$, hexagonal $(\beta)$, and triclinic $(\gamma) \cdot{ }^{10} \alpha$ Modification is most frequently formed during the crystallization of industrial i-PP grades, although $\beta$-modification can also appear at higher undercooling. ${ }^{11}$ The $\gamma$-modification is observed in degraded, low molecular weight PP or when it crystallizes under high pressure. Crystallization of iPP can be described by the Avrami expression over a wide crystallization temperature range, ${ }^{11}$ and Avrami exponents between $n=1$ and $n=4$ are determined by DSC and depolarization microscopy, ${ }^{12-22}$ usually explained by different nucleating mechanisms.

The melting of i-PP is affected by many factors, such as molecular mass and molecular mass distribution, degree of isotacticity, and head-to-tail sequences and the presence of different crystal forms. ${ }^{23}$ The appearance of multiple peaks during the melting, observed by DSC analysis, is usually related to some of the following factors: different crystal structures, phenomenon of recrystallization and perfection during fusion, and different crystal sizes. Besides the fact that the melting and crystallization behavior of i-PP are comprehensively reviewed, ${ }^{10}$ some peculiarities are still a subject of debate.

This article is part of a study of model and bulk composites, based on i-PP and glass (or carbon) fibers, produced from knitted textile preforms of hybrid yarns. ${ }^{24}$ First, we report the results on crystallization and fusion of textile grade i-PP, used for the processing of hybrid yarns and the corresponding knitted fabrics.

\section{THEORETICAL BACKGROUND}

The crystallization kinetics of polymeric materials under isothermal conditions for various modes 

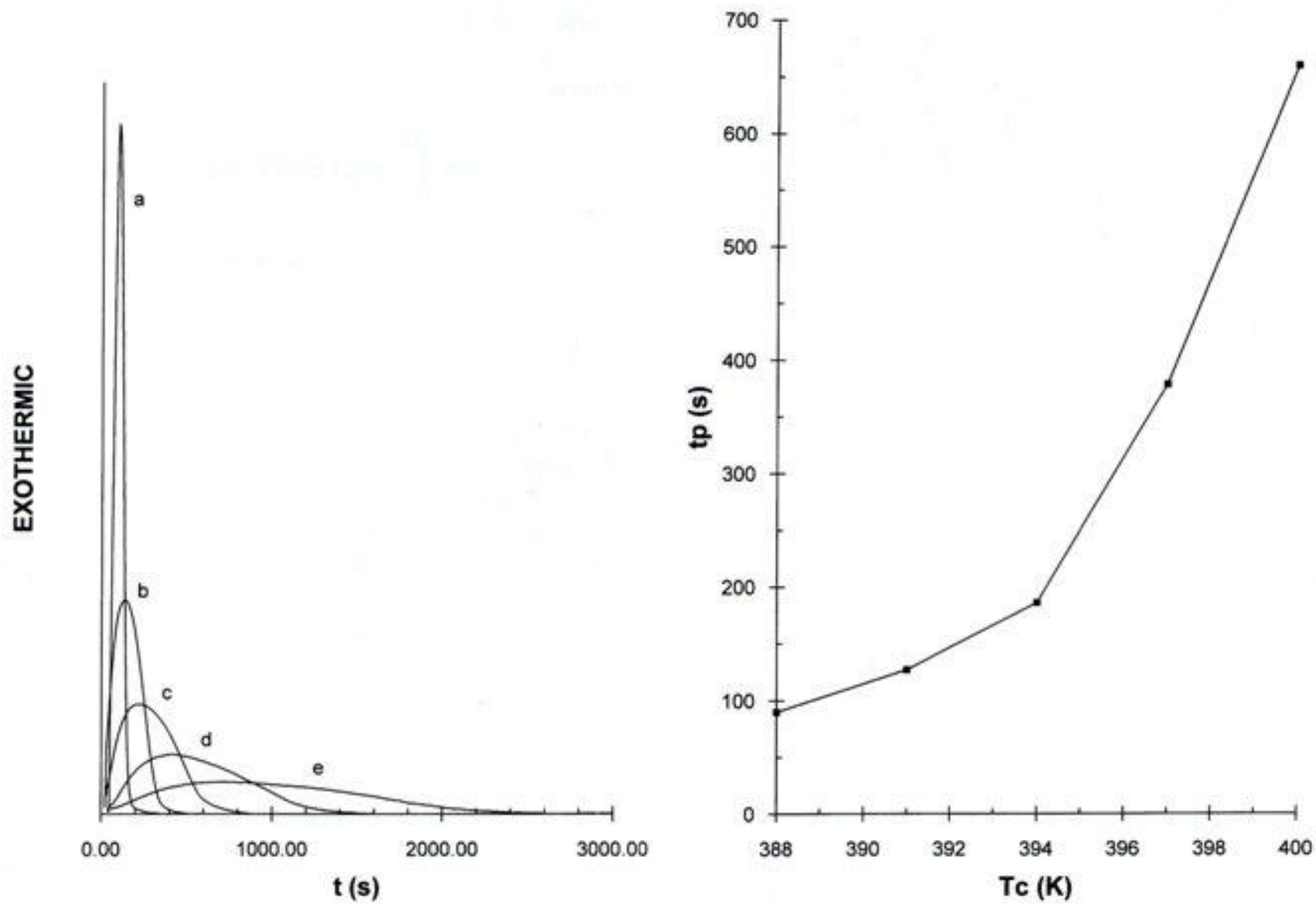

Figure 2 (a) DSC thermograms of isothermal crystallization at different $T_{c}$ : (a) 388 K; (b) $391 \mathrm{~K}$; (c) $394 \mathrm{~K}$; (d) $397 \mathrm{~K}$; (e) $400 \mathrm{~K}$. (b) $t_{p}$ as a function of $T_{c}$.

of nucleation and growth can be well approximated by the known Avrami ${ }^{25}$ equation. This equation is derived by assuming random nucleation, a constant growth rate, and a constant rate of nucleation (or a constant nucleation density), and the general form of the Avrami expression is given as

$$
\alpha=1-\exp \left(-K t^{n}\right)
$$

where $\alpha$ is extent of crystallization (crystal conversion); $n$, the Avrami exponent; and $K$, a rate constant which usually follows the Arhenius relationship with temperature:

$$
K=A \exp (E / R T)
$$

where $A$ is the preexponential factor, and $E$, the activation energy. The value of the Avrami exponent depends on the mechanism of nucleation and the geometry of crystal growth, and the constant $K$ includes nucleation parameters as well as growth-rate parameters. DSC is the most widely used experimental technique, enabling isothermal crystallization as well as kinetic studies. The extent of crystallization as a function of time and/ or temperature can be recorded and the parameters of crystallization determined.

In nonisothermal DSC studies, a polymer sample is usually crystallized by cooling $(\beta<0)$ from the melt or by heating $(\beta>0)$ from an amorphous state under the influence of a linear temperature change ( $T=T_{0}+\beta t$, where $T_{0}$ is the initial temperature; $\beta$, the scanning rate; and $t$, the time). Under such conditions, the crystallization process may be treated as a sequence of infinitesimally small isothermal crystallization steps. ${ }^{26,27}$ Hence, the rate equation for the isothermal condition:

$$
\alpha=d \alpha / d t=n K t^{n-1}(1-\alpha)
$$

should also be applicable for the nonisothermal condition and has the form

$$
\alpha=d \alpha / d t=n K\left[\left(T-T_{0}\right) / \beta\right](1-\alpha)
$$

Integration of eq. (4) leads to 


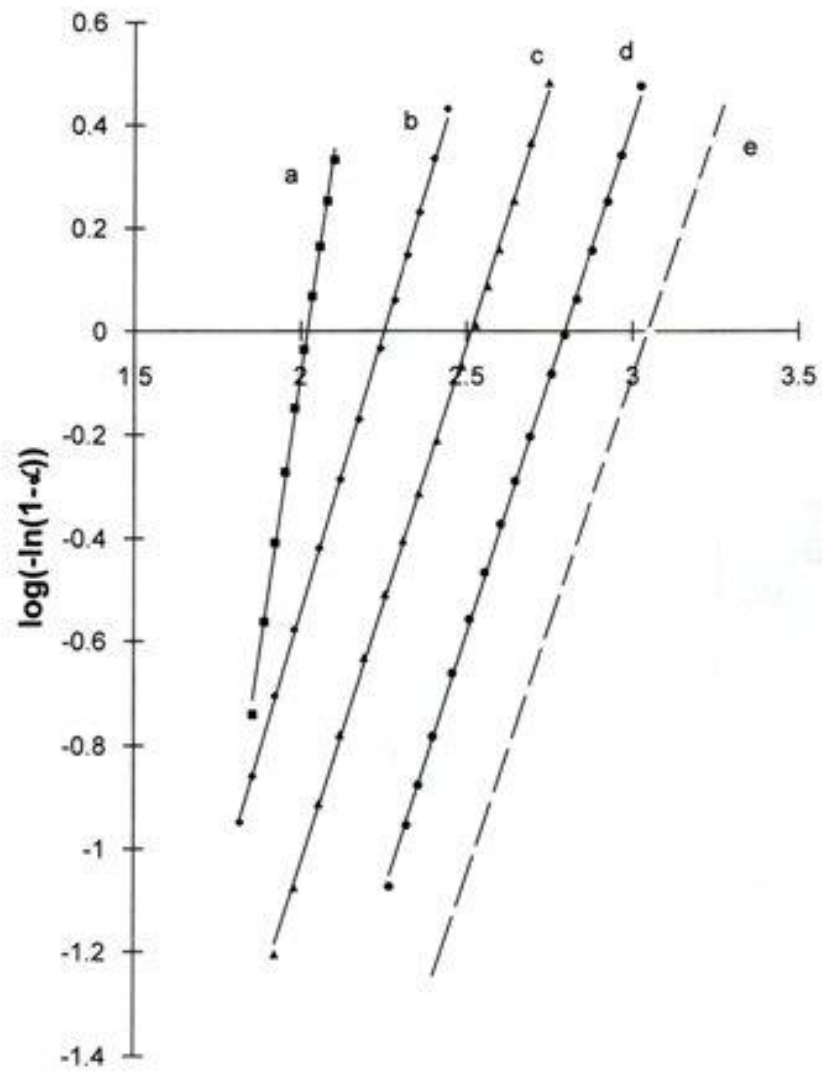

Figure 3 Avrami plots of isothermal crystallization of i-PP at different $T_{c}$ : (a) $388 \mathrm{~K}$; (b) $391 \mathrm{~K}$; (c) $394 \mathrm{~K}$; (d) $397 \mathrm{~K}$; (e) $400 \mathrm{~K}$.

$$
\alpha=1-\exp \left(-\int_{t_{0}}^{t} n K t^{n-1} d t\right)
$$

Equation (5) was derived by Ozawa from fundamental consideration in order to describe the nonisothermal crystallization, and this method is the most commonly used to analyze the nonisothermal kinetics data. ${ }^{28}$ Equation (5) can be expressed as

$$
\log [-\ln (1-\alpha)]=\log [\chi(T)]-n \log (\beta)
$$

where

$$
\chi(T)=n A \int_{T_{0}}^{T} \exp (E / R T)\left(T-T_{0}\right)^{n-1} d T
$$

Ozawa suggested that the Avrami exponent can be obtained by plotting $\log [-\ln (1-\alpha)]$ vs. $\log (\beta)$ at constant temperature. The Ozawa approach is valid only when a linear dependence of $\log [-\ln (1$ $-\alpha)]$ vs. $\log \beta$ exists.

The method developed by Harnisch and Muschik $^{29}$ is derived from the Avrami eq. (1) and allows determination of the Avrami exponent during nonisothermal crystallization of polymers. Assuming that the enthalpy of transformation is independent of temperature and rearranging eq. (1), the following equation is obtained:

$$
\begin{aligned}
& n=1+\left(\operatorname { l n } \left(\alpha_{i} /\left(1-\alpha_{i}\right)\right.\right. \\
&-\ln \left[\alpha_{j} /\left(1-\alpha_{j}\right)\right] / \ln \left(\beta_{j} / \beta_{i}\right) \\
& \quad\left|\beta_{j}\right|<\left|\beta_{i}\right|, \quad T=T_{c}
\end{aligned}
$$

To determine the values of the exponent by this method, it is necessary to record nonisothermal crystallization thermograms at least at two different cooling rates.

\section{EXPERIMENTAL}

The kinetics of the crystallization process, in the dynamic and isothermal regime, was followed using a Perkin-Elmer DSC-7 analyzer. The experiments were carried out under nitrogen, and the calibration was performed with indium and zinc. To analyze the effect of the cooling rate on the crystallization kinetics, PP samples (3-5 mg;

Table I The Isothermal Crystallization Data for i-PP

\begin{tabular}{ccccc}
\hline $\begin{array}{c}T_{c} \\
(\mathrm{~K})\end{array}$ & $\begin{array}{c}\Delta H_{m}{ }^{\mathrm{a}} \\
(\mathrm{kJ} / \mathrm{mol})\end{array}$ & $\begin{array}{l}X_{c}{ }^{\mathrm{b}} \\
(\%)\end{array}$ & $n$ & $\begin{array}{c}K \\
\left(\mathrm{~s}^{-1}\right)\end{array}$ \\
\hline 388 & 3.58 & 41.0 & 4.39 & $1.35 \times 10^{-6}$ \\
391 & 3.60 & 41.4 & 2.18 & $1.22 \times 10^{-6}$ \\
394 & 3.67 & 42.2 & 2.01 & $8.86 \times 10^{-6}$ \\
397 & 4.25 & 48.9 & 2.00 & $2.58 \times 10^{-6}$ \\
400 & 3.96 & 45.5 & 1.93 & $1.27 \times 10^{-6}$ \\
\hline
\end{tabular}

" $\Delta H_{m}$, heat of fusion after isothermal crystallization of i-PP performed at given $T_{c}$.

${ }^{\mathrm{b}} X_{c}$, degree of crystallinity determined as $X_{c}=\Delta H_{c} / \Delta H_{c}^{*} \cdot 100 \% . \Delta H_{c}^{*}=8.7 \mathrm{~kJ} / \mathrm{mol}$. 


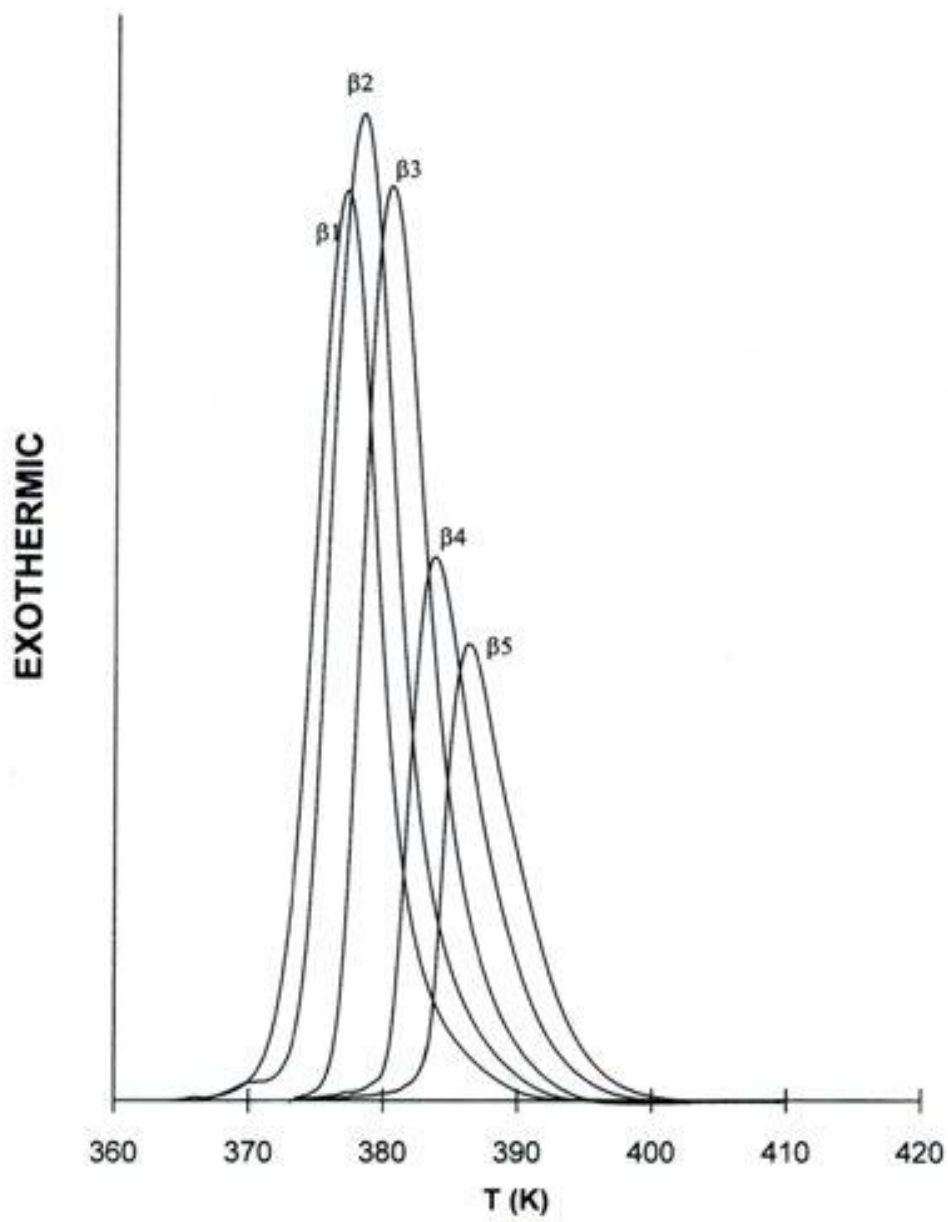

Figure 4 DSC thermograms of nonisothermal crystallization of i-PP at different cooling rates: $\beta 1=20 \mathrm{~K} / \mathrm{min} ; \beta 2=15 \mathrm{~K} / \mathrm{min} ; \beta 3=10 \mathrm{~K} / \mathrm{min} ; \beta 4=5 \mathrm{~K} / \mathrm{min} ; \beta 5=3 \mathrm{~K} /$ min.

DAPLEN MT55-Prespatex, $\mathrm{MFI} / 230^{\circ} \mathrm{C}=12-14$ $\mathrm{g} / 10 \mathrm{~min}$ ) were heated at $10 \mathrm{~K} / \mathrm{min}$ and then cooled at different cooling rates $(3,5,10,15$, and $20 \mathrm{~K} / \mathrm{min}$ ). At an isothermal regime, PP samples were heated to $478 \mathrm{~K}$ (heating rate $80 \mathrm{~K} / \mathrm{min}$ ),

\section{Table II Nonisothermal Crystallization Data} for i-PP

\begin{tabular}{ccccc}
\hline$N^{\mathrm{n}}$ & $\begin{array}{c}-\beta \\
(\mathrm{K} / \mathrm{min})\end{array}$ & $\begin{array}{c}T_{c} \\
(\mathrm{~K})\end{array}$ & $\begin{array}{c}-\Delta H_{\mathrm{c}} \\
(\mathrm{kJ} / \mathrm{mol})\end{array}$ & $\begin{array}{l}X_{c}^{\mathrm{b}} \\
(\%)\end{array}$ \\
\hline 1 & 20 & 377.3 & 3.84 & 38.0 \\
2 & 15 & 378.3 & 3.54 & 40.7 \\
3 & 10 & 380.5 & 3.64 & 41.8 \\
4 & 5 & 383.8 & 3.65 & 42.0 \\
5 & 3 & 386.3 & 3.70 & 42.6 \\
\hline
\end{tabular}

${ }^{a}$ Number of experiment.

${ }^{\mathrm{b}} X_{\varepsilon}$, degree of crystallinity determined as $X_{\varepsilon}=\Delta H_{c} l$ $\Delta H_{c}^{*} \cdot 100 \% . \Delta H_{e}^{*}=8.7 \mathrm{~kJ} / \mathrm{mol}$. held at the molten state for $5 \mathrm{~min}$ to erase the former thermal history, and then rapidly cooled at the maximum cooling rate $(200 \mathrm{~K} / \mathrm{min})$ to the crystallization temperature, $T_{c} . T_{c}$ was then held constant until crystallization was completed. The equilibrium melting point of PP, $T_{m}^{0}$, was determined by the Hoffman-Weeks method, ${ }^{7}$ extrapolating the experimental points of the plot $T_{m}$ $=f\left(T_{c}\right)$ to the intercept with the plot $T_{m}=T_{c}$.

\section{RESULTS AND DISCUSSION}

\section{Crystallization}

The percent of isotactic content in PP was calculated from the FTIR spectra ${ }^{30}$ by the equation

$$
\begin{aligned}
& \% \text { iso } \\
& \qquad=[1-(K-1) /(0.853 K-0.113)] \times 100
\end{aligned}
$$




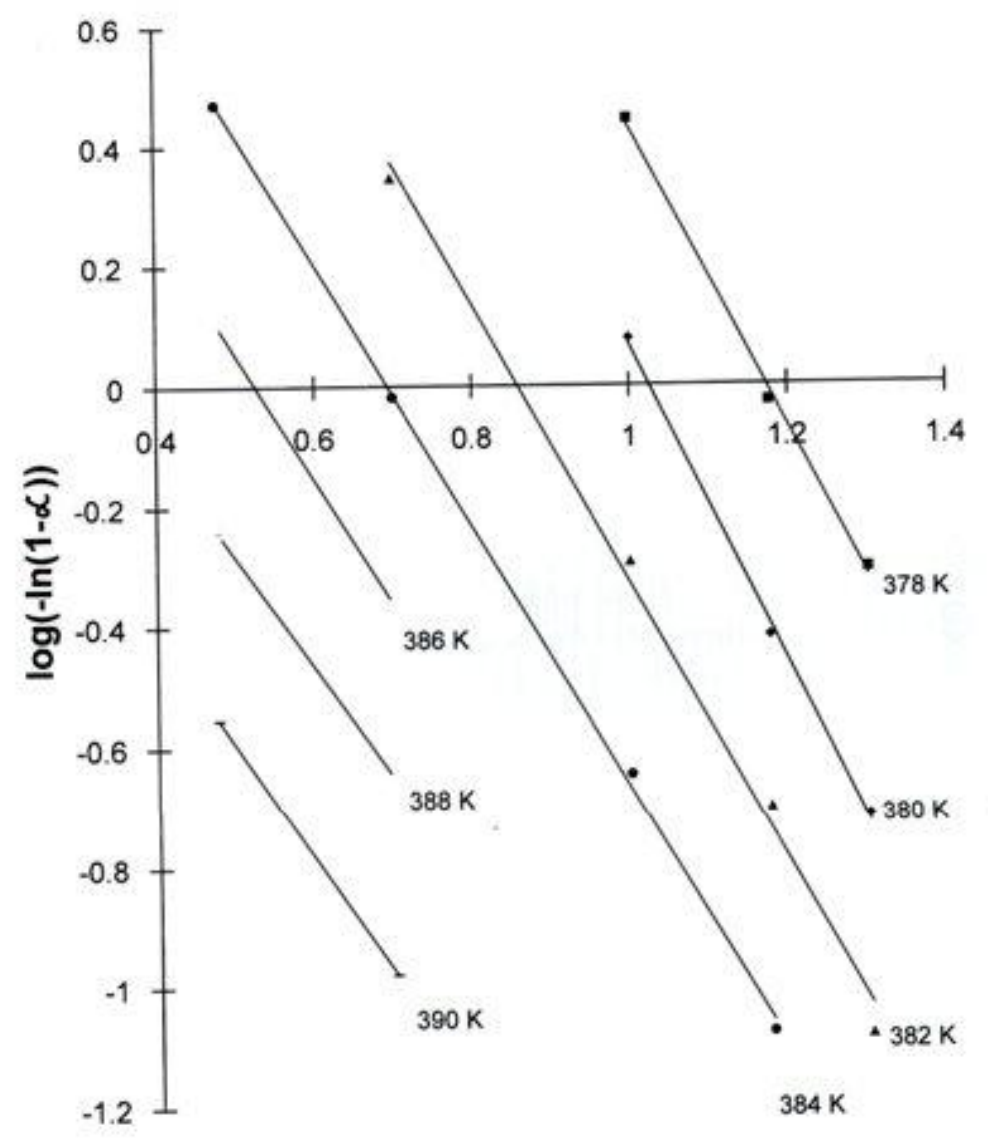

$\log (\beta)$

Figure 5 Ozawa plots of $\log \{\ln [1-\alpha]\}$ against $\log \left(\beta_{t}\right)$ from the data presented in Figure 4.

where $K=A_{974} / A_{995} . A_{974}$ and $A_{995}$ are absorbances of the bands at 974 and $995 \mathrm{~cm}^{-1}$, respectively, determined from curve-fitted FTIR spectra, recorded on Perkin-Elmer FTIR-2000 spectrometer. The i-PP content was $78 \%$.

The diffraction pattern recorded on i-PP film (Universal X-ray diffractometer, $\mathrm{KCu} \alpha, 40 \mathrm{kV}, 20$ $\mathrm{mA}$ ) is represented in Figure 1, and the percent of crystallinity calculated by the Hermans-Weidinger method ${ }^{31}$ was 64 . The results of isothermal DSC experiments are presented in Figure 2, and the kinetic parameters were inferred from the graphic representation of the Avrami equation (Fig. 3). As can be seen, the crystallization below $390 \mathrm{~K}$ proceeds too fast (see Fig. 2 and Table I), and it is also assumed that a considerable amount of crystallinity can be produced already during the cooling from the melt to the $T_{c}{ }^{26}$ This is confirmed by investigation of the melting behavior of isothermally crystallized i-PP (see Fig. 8) and the appearance of two melting peaks in the DSC curve.
The time required to reach the exothermal DSC peak, $t_{p}$, [Fig. 2(b)] increases with increasing crystallization temperature. These results are in agreement with the common behavior of crystallization rates at moderate undercooling. ${ }^{26}$

The values of the Avrami exponents are presented in Table I. For the studied interval of crystallization temperatures (388-400 K), different values for $n$, ranging from 1.93 to 4.39 , were found. The values of the Avrami exponent depends on the applied experimental method (dilatometry, DSC, optical microscopy). ${ }^{32,33}$ DSC data for the Avrami exponent varied with the temperature range: 2.0 $(390-413 \mathrm{~K}),{ }^{12,13} 2.7-3.5(383-403 \mathrm{~K}),{ }^{14,15} 2.0$ $2.6(396-407 \mathrm{~K}),{ }^{16} \sim 3.0(387-405 \mathrm{~K}),{ }^{17}$ and 5.9 $7.6(403-413 \mathrm{~K}){ }^{18}$ Dilatometry-based data are usually close to $n=3(396-429 \mathrm{~K})^{19-21}$ and $2.8-$ $4.0(403-433 \mathrm{~K}){ }^{22}$ Lower values (in the range of $0.5-1.6$ ) for $n$ were found in the presence of unmelting materials. ${ }^{13}$ According to Godovsky and Slonim$\mathrm{sky},{ }^{13}$ this discrepancy in the data is connected with the morphology of the polymer crystallized from the 


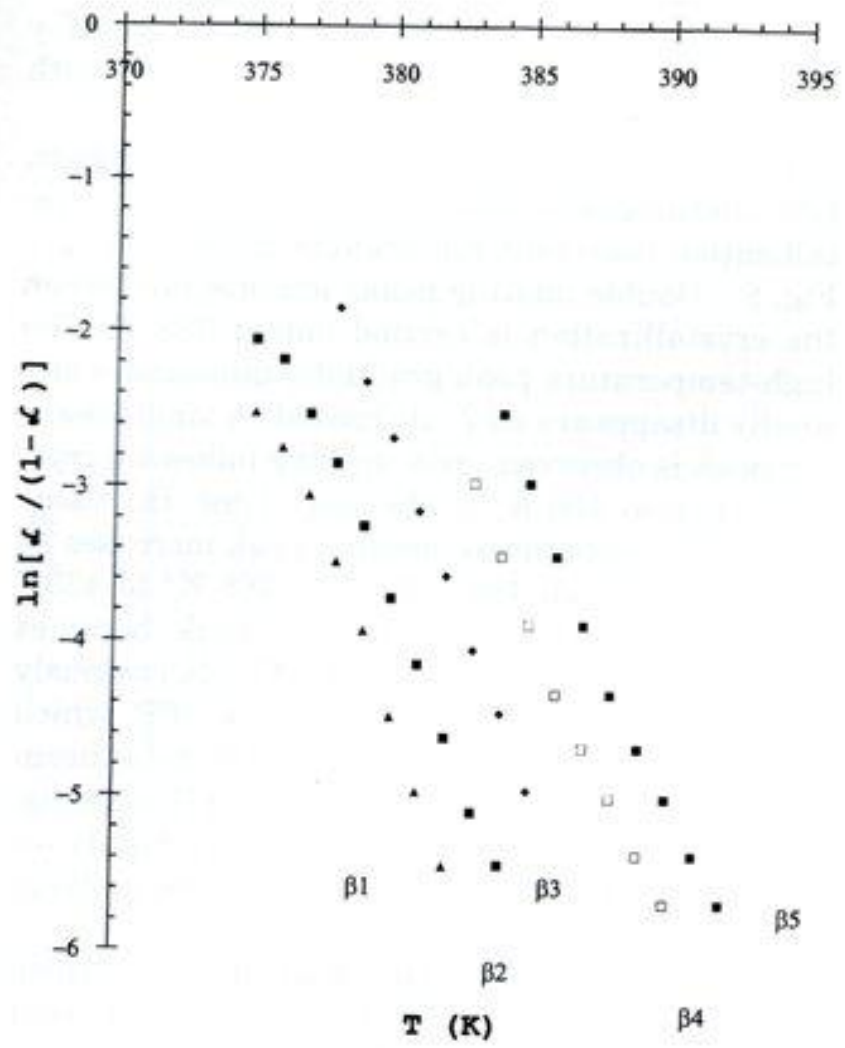

Figure 6 Harnisch-Muschik plot of $\ln [\alpha /(1-\alpha)]$ against temperature at different cooling rates $(\beta)$, listed in Table II.

melt, and a general conclusion is derived: that increasing $n$ is related to several factors, such as decreasing of supercooling, changes from athermal to thermal nucleation, and initiation of the secondary crystallization processes. ${ }^{33}$

Variation of the rate constant, $K$, in the range of $1.35 \times 10^{-9}(388 \mathrm{~K})$ to $8.86 \times 10^{-6}(394 \mathrm{~K})$ confirmed that the rate constant is in a complex relation to the temperature. Compared to the literature data for $K$ at the same crystallization temperatures, ${ }^{13}$ our data are lower. Based on the obtained results, it can be proposed that the mechanism of nucleation is homogenic at lower crystallization temperatures and heterogenic at higher temperatures. From the practical point of view, study of the kinetics of crystallization in a nonisothermal regime is, further, more important, since the processing of thermoplastic polymers and composites is usually carried out under nonisothermal conditions.

The DSC scans of i-PP recorded during cooling from the melt $(478 \mathrm{~K})$ at different cooling rates are shown in Figure 4. The peak crystallization temperature, $T_{c}$ (corresponding to temperature at which the maximum crystallization rate is reached), changes from 377.3 to $386.3 \mathrm{~K}$ as the cooling rate decreases from 20 to $3 \mathrm{~K} / \mathrm{min}$ (Table II). A slightly higher degree of crystallinity is also found for the lower cooling rates, obviously due to the formation of a more ordered crystalline structure.

An Ozawa plot, obtained by using data for $-\beta$ $=20,15,10,5$, and $3 \mathrm{~K} / \mathrm{min}$, for different crystallization temperatures, is shown in Figure 5, and a value of 2.33 for the Avrami exponent was determined based on these results, which is in agreement with the results from isothermal crystallization at $391-400 \mathrm{~K}$, reported in Table I. The derived values for an exponent determined by the Harnisch-Muschik method, listed in Figure 6, are $n_{1-2}=3.59, n_{2-3}=3.47, n_{3-4}=2.52$, and $n_{4-5}$, $=2.46$.

A similar trend in the results of isothermal and nonisothermal crystallization analyzed by this method can be observed. The lower values for $n$ found for lower cooling rates are possibly due to a predominant homogeneous mechanism in spite of a heterogeneous mechanism at higher cooling rates.

\section{Melting Behavior}

The equilibrium melting temperature of $\mathrm{i}-\mathrm{PP}$ was determined by applying the Hoffman-Weeks

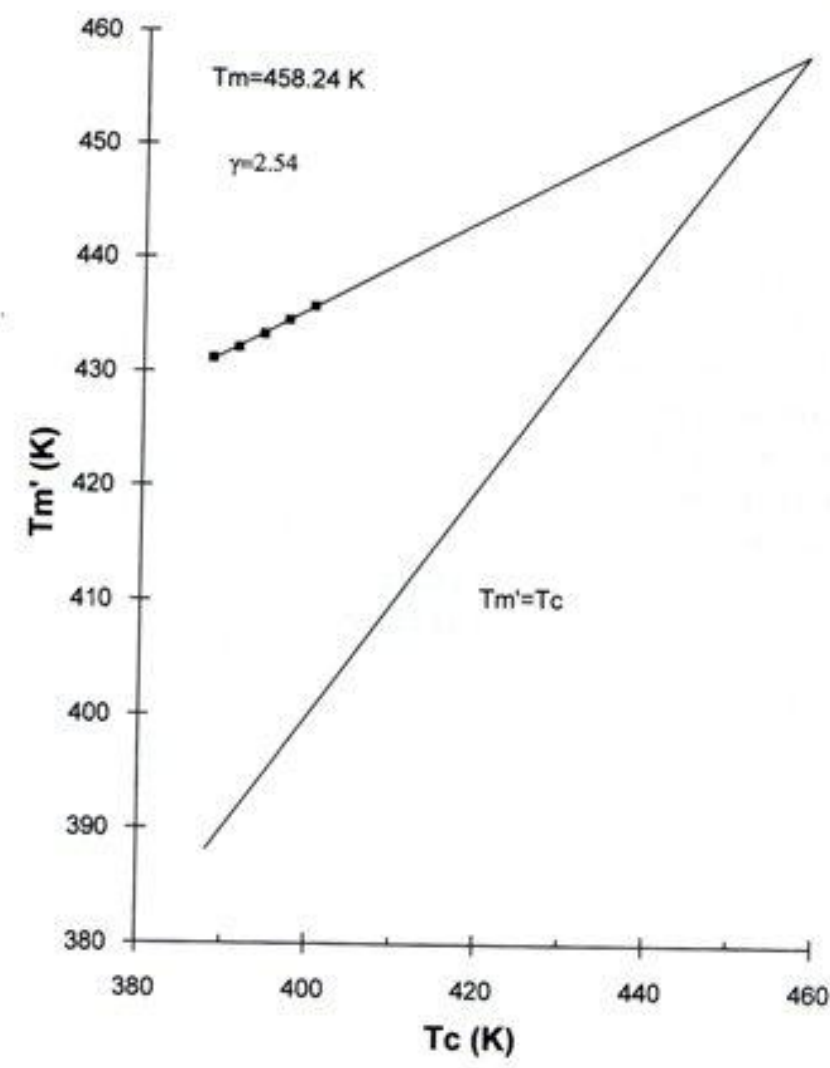

Figure 7 Hoffman-Weeks plot for i-PP. 


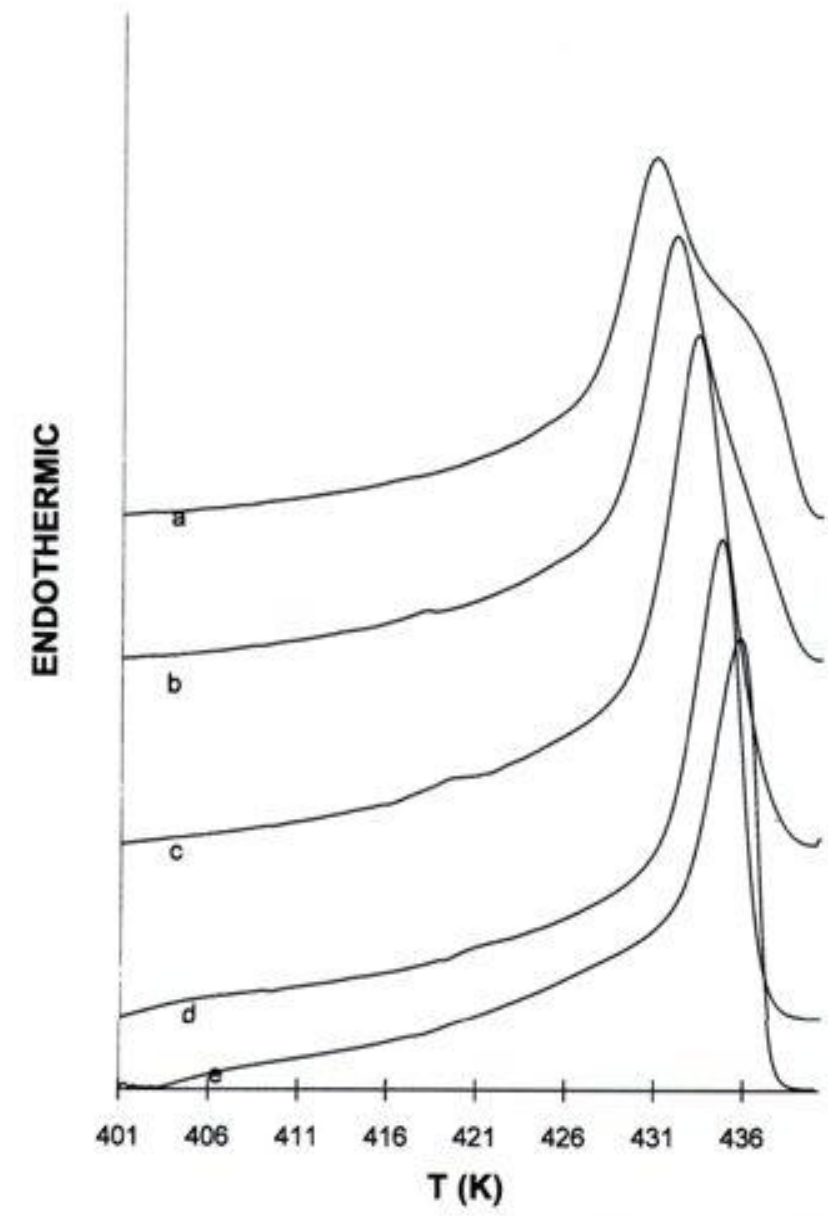

Figure 8 DSC thermograms of melting of i-PP after isothermal crystallization at different $T_{\mathrm{e}}$ : (a) $388 \mathrm{~K}$; (b) $391 \mathrm{~K}$; (c) $394 \mathrm{~K}$; (d) $397 \mathrm{~K}$; (e) $400 \mathrm{~K}$ (heating rate $10 \mathrm{~K} / \mathrm{min}$ ).

method, by plotting melting temperatures, $T_{m}$, against isothermal crystallization temperatures, $T_{c}$, as presented in Figure 7. The studied i-PP sample was first crystallized isothermally (as reported in the Experimental part), and then, following each crystallization process, the solidified sample was heated from $T_{c}$ to $478 \mathrm{~K}$ at a heating rate of $10 \mathrm{~K} / \mathrm{min}$. The polymer melting temperature increases linearly as crystallization temperature is increased.

Using the Hoffman-Weeks relation, the constant $\gamma$ which represents the ratio between the final thickness of the crystalline lamellae and the initial critical thickness, can be determined from the equation ${ }^{7}$

$$
T_{m}^{\prime}=T_{m}^{0}(\gamma-1 / \gamma)+T_{c} / \gamma
$$

where $T_{m}^{\prime}$ is the observed melting temperature; $T_{m}^{0}$, the equilibrium melting temperature; and $T_{c}$, the crystallization temperature. The value of $\gamma$ $=2.54$ is found, which is in good agreement with the literature data for i-PP. ${ }^{7}$

Depending on the crystallization temperature, DSC thermograms obtained after isothermal crystallization represent remarkable differences (see Fig. 8). Double melting peaks are observed when the crystallization is carried out at $388 \mathrm{~K}$. The high-temperature peak gradually diminishes and finally disappears as $T_{c}$ increased. A single melting peak is observed upon melting following crystallization at $400 \mathrm{~K}$. At the same time, the value of the low-temperature melting peak increases as $T_{c}$ increases from $430.7 \mathrm{~K}\left(T_{c}=388 \mathrm{~K}\right)$ to 435.7 $\left(T_{c}=400 \mathrm{~K}\right)$ and the melting peak becomes sharper. The shoulder peak at $435.7 \mathrm{~K}$ obviously indicates the melt reorganization of i-PP, which may include an increasing order in crystal spherulites, interfibrilar growth, and crystal thickening. Crystals formed at higher $T_{c}$ are more highly ordered, and, therefore, only a few of them undergo recrystallization during melting.

The DSC melting thermograms performed after nonisothermal crystallization at different

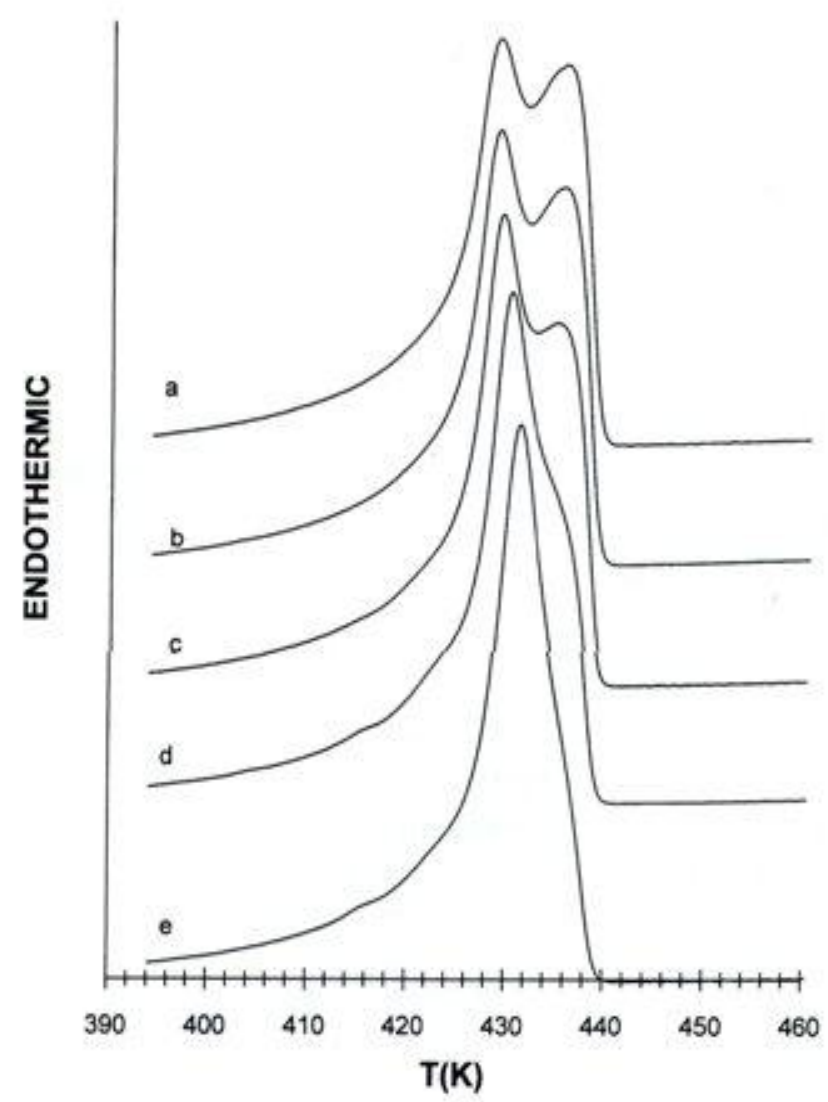

Figure 9 DSC melting thermograms after nonisothermal crystallization at different cooling rates: (a) $\beta_{1}$; (b) $\beta_{2}$; (c) $\beta_{3}$; (d) $\beta_{4}$; (e) $\beta_{5}$. 


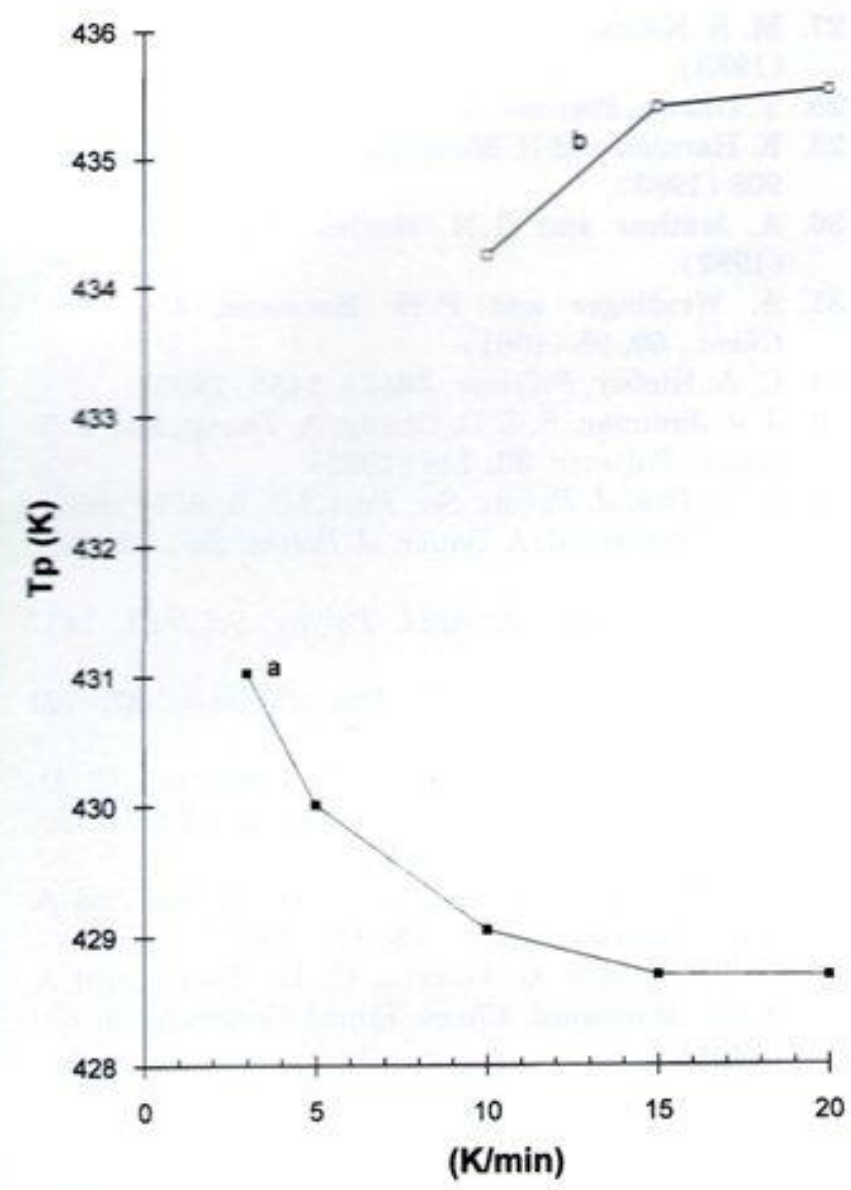

Figure 10 Changes of melting peak temperature registered at different cooling rates: (a) low-temperature melting peak; (b) high-temperature melting peak.

cooling rates are presented in Figure 9. One can see multiple peaks, attributed to such factors as two different crystal structures, different crystal sizes, and perfection during heating. ${ }^{34-37}$ Petracone et al. reported that the double melting peaks may be attributed to existing of less-ordered $\alpha_{1}$ and more-ordered $\alpha_{2}$ forms with a well-defined deposition of up and down helices in the unit cells. ${ }^{38-40}$ Using SAX and DSC techniques, the existence of two phases, $\alpha 1$ and $\alpha 2$, was confirmed, and the Avrami exponent for both crystallization phases was calculated. ${ }^{33}$

Obviously, polypropylene crystallized during nonisothermal cooling at a lower rate $(3 \mathrm{~K} / \mathrm{min}$ ) exhibits a single peak due to the more-ordered structure, compared to samples cooled at higher rates. Figure 10 confirms that during the nonisothermal crystallization at higher cooling rates two crystal phases exist, with corresponding melting temperatures: $\alpha 1 / T_{m}=430 \mathrm{~K}$ and $\alpha 2 / T_{m}$ $=435 \mathrm{~K}$.

\section{CONCLUSION}

The crystallization and melting behavior of textile-grade i-PP were studied by DSC. Isothermal and nonisothermal crystallization data were analyzed by the Avrami, Ozawa, and HarnischMuschik methods. Isothermal crystallization was carried out at $389-400 \mathrm{~K}$, and values for the Avrami exponent ranging from 1.93 to 4.39 were determined. Double melting peaks were observed upon isothermal crystallization carried out at lower $T_{c}$, while a single melting peak appeared after isothermal crystallization at $400 \mathrm{~K}$. During nonisothermal crystallization, it was found that $T_{\mathrm{c}}$ changes from 377 to $386 \mathrm{~K}$ as the cooling rate decreases from 20 to $3 \mathrm{~K} / \mathrm{min}$. Double melting peaks were also observed upon nonisothermal crystallization performed at higher cooling rates. Similar values for $n$ were determined both by Avrami's and Ozawa's methods (nonisothermal regime), and a similar trend, confirmed by the Harnisch-Muschik approach, was observed for isothermal and nonisothermal crystallization, attributed to a predominant homogeneous mechanism of nucleation at lower cooling rates (or lower isothermal $T_{c}$ ) in spite of a heterogeneous one at higher cooling (or higher isothermal $T_{c}$ ).

\section{REFERENCES}

1. B. Wulfhorst, G. Tetzlaff, and R. Kaldenhoff, Chem. Text., 42(3), 10 (1992).

2. K. Horsting, B. Wulfhorst, G. Franzke, and P. Offermann, SAMPE J., 29, 7 (1993).

3. E. Mader, G. Hofmann, and U. Engelmann, Techtext. Symp., 327, 1 (1994).

4. D. Cutolo and A. Savadori, Polym. Adv. Tech., 5, 545 (1994).

5. S. Ramakrishna, H. Hamada, N. K. Cnong, and Z. Maekawa, in ICCM-10 Proceed IV-245, Whistler, BC, Canada, Aug. 1995.

6. F. Hoecker and J. Karger-Kocsis, J. Adhes., 52, 81 (1995).

7. M. Avella, E. Martuscelli, C. Selliti, and E. Caragnani, J. Mater. Sci., 22, 3185 (1987).

8. N. Klein, D. Selivansky, and G. Marom, Polym. Compos., 16(3), 189 (1995).

9. J. L. Thompson and A. A. Van Rooyen, J. Mater. Sci., 27, 889 (1992).

10. J. Karger-Kocsis and J. Varga, Polypropylene: Structure, Blends and Composites, Vol. 1, Structure and Morphology, Chapman \& Hall, London, 1995.

11. B. Lotz and C. Wittmann, Progr. Colloid Polym. Sci., 87, 3 (1992). 
12. Y. K. Godovsky and G. L. Slonimsky, Vysokomol. Soedin., 8, 403 (1966).

13. Y. K. Godovsky and G. L. Slonimsky, J. Polym. Sci. Phys. Ed., 12, 1053 (1974).

14. H. Heyns and S. Heyez, Proc. Third. Int. Conf. Therm. Anal., 3, 341 (1971).

15. C. F. Pratt and S. Y. Hobbs, Polymer, 17, 12 (1976).

16. M. Avella, E. Martuscelli, and M. Pracella, J. Therm. Anal., 28, 237 (1983).

17. H. J. Tai, W. Y. Chiu, L. W. Chen, and L. H. Chu, J. Appl. Polym. Sci., 42, 3111 (1991).

18. R. Paiz, P. Nigam, and R. D. Wesson, SPE ANTEC Tech. Pap., 39, 242 (1993).

19. B. V. Falkai and H. A. Stuart, Z. Kolloid, 162, 138 (1959).

20. L. Marker, P. M. Hay, G. P. Toilley, R. M. Early, and O. J. Sweeting, J. Polym. Sci., 38, 33 (1959).

21. J. H. Griffith and B. G. Ranby, J. Polym. Sci., 38, 107 (1959).

22. P. Parrini and G. Corrieri, Makromol. Chem., 62, 83 (1963).

23. J. Varga, J. Mater. Sci., 27, 2557 (1992).

24. G. Dembovski, A. Grozdanov, G. Bogoeva-Gaceva, K. Ljapceva, and B. Mangovska, Textil, 45, 11 (1996).

25. M. Avrami, J. Chem. Phys., 9, 177 (1941).

26. A. Ziabicki, Appl. Polym. Symp., 6, 1 (1967).
27. M. R. Kamal and E. Chu, Polym. Eng. Sci., 23, 27 (1983).

28. T. Ozawa, Polymer, 12, 150 (1971).

29. K. Harnich and H. Muschik, Coll. Polym. Sci., 261, 908 (1983).

30. A. Mathur and G. N. Mathur, Polymer, 23, 54 (1982).

31. A. Weidinger and P.H. Hermans, Makromol. Chem., 50, 98 (1961).

32. C. A. Hieber, Polymer, 36( 7), 1455 (1995).

33. J. J. Janimac, S. Z. D. Cheng, A. Zhang, and E. T. Hsieh, Polymer, 33, 728 (1992).

34. K. D. Pae, J. Polym. Sci. Part A-2, 6, 657 (1968).

35. K. D. Pae and J. A. Sauer, J. Polym. Sci., 12, 1908 (1968).

36. R. J. Samuels, J. Appl. Polym. Sci., 13, 1417 (1975).

37. Y. S. Yadav and P. C. Join, Polymer, 27, 721 (1986).

38. G. Guerra, V. Petraccone, P. Corradini, C. De Rossa, R. Napolitano, and B. Pirozzi, J. Polym. Sci. Polym. Phys, Ed., 22, 1029 (1984).

39. V. Petraccone, G. Guerra, C. De Rossa, and A. Tuzu, Macromolecules, 18, 813 (1985).

40. V. Petraccone, G. Guerra, C. De Rossa, and A. Tuzu, Macromol. Chem. Rapid Commun., 5, 631 (1984). 